\title{
Pneumatosis Cystoides Intestinalis Identified on Screening Colonoscopy With Associated Pneumoperitoneum
}

\author{
Matthew J. Lommen ${ }^{1}$, Omar Zineldine ${ }^{1}$, Tej I. Mehta ${ }^{1}$, Logan E. Radtke ${ }^{2}$, Oluwagbenga Serrano ${ }^{3}$ \\ 1. Radiology, University of South Dakota Sanford School of Medicine, Sioux Falls, USA 2. Surgery, University of South \\ Dakota Sanford School of Medicine, Sioux Falls, USA 3. Gastroenterology, Good Samaritan Hospital, Vincennes, USA
}

Corresponding author: Matthew J. Lommen, matthew.lommen@coyotes.usd.edu

\begin{abstract}
Pneumatosis cystoides intestinalis (PCI) is defined by the presence of gas within the bowel wall. It is often asymptomatic and usually benign but may be associated with significant morbidity and mortality. In this patient, PCI was found incidentally on screening colonoscopy, and biopsy of the affected mucosa resulted in deflation of a cyst. Pneumoperitoneum was then identified on subsequent CT. Because pneumoperitoneum is associated with bowel perforation in most cases, it is often treated as an indication for operation. This case of benign and asymptomatic pneumoperitoneum was managed conservatively without complications. Clinicians should be able to identify PCI as a potentially benign finding on colonoscopy as well as a potentially benign cause of pneumoperitoneum. This understanding presents an opportunity to avoid the unnecessary morbidity and costs associated with surgical exploration or additional endoscopic procedures.
\end{abstract}

Received 06/26/2020 Review began $07 / 07 / 2020$ Review ended 07/25/2020 Published 08/01/2020

(c) Copyright 2020 Lommen et al. This is an open access article distributed under the terms of the Creative Commons Attribution License CC-BY 4.0., which permits unrestricted use, distribution, and reproduction in any medium, provided the original author and source are credited.
Categories: Internal Medicine, Gastroenterology, General Surgery

Keywords: pneumatosis cystoides intestinalis, colonoscopy, pneumoperitoneum, pneumatosis intestinalis

\section{Introduction}

Pneumatosis cystoides intestinalis (PCI) is a rare finding of intramural gas within the walls of the small and/or large intestine. PCI is not itself a diagnosis but is a radiographic or physical finding that may be associated with an underlying disease process [1]. An autopsy series reported a prevalence of $0.03 \%$ in the general population, though the true prevalence of PCI remains unknown [2]. It may be associated with severe disease such as necrotizing enterocolitis or identified incidentally on radiographic, endoscopic, or pathologic examination [2,3]. Rarely, PCI represents a cause of benign pneumoperitoneum or air within the peritoneal cavity [4]. Despite current knowledge and hypotheses regarding the pathogenesis, epidemiology, presentation, diagnosis, and management of PCI, there remains a lack of multicenter, prospective clinical trials assessing factors contributing to diagnosis and management of benign versus pathologic PCI.

We present a case of PCI identified incidentally on screening colonoscopy with concurrent pneumoperitoneum present on CT. Our objective is a contribution to, and review of, current literature regarding this topic.

\section{Case Presentation}

A 65-year-old woman presented for her first screening colonoscopy. Her medical history was significant for morbid obesity, anxiety, hypertension, and a 46 pack-year history of tobacco use. She had previously undergone a laparoscopic cholecystectomy, ventral hernia repair, and cylindroma excision but had no surgeries in the 12 months prior to her presentation. Her medications at the time included hydrochlorothiazide and losartan. She had no family history of colon cancer or any recent significant gastrointestinal symptoms.

The patient's colonoscopy revealed polypoid grape-like masses protruding through the mucosa, consistent with PCI at the splenic flexure as well as small, benign colonic polyps at other areas of the colon (Figure 1). 


\section{Cureus}

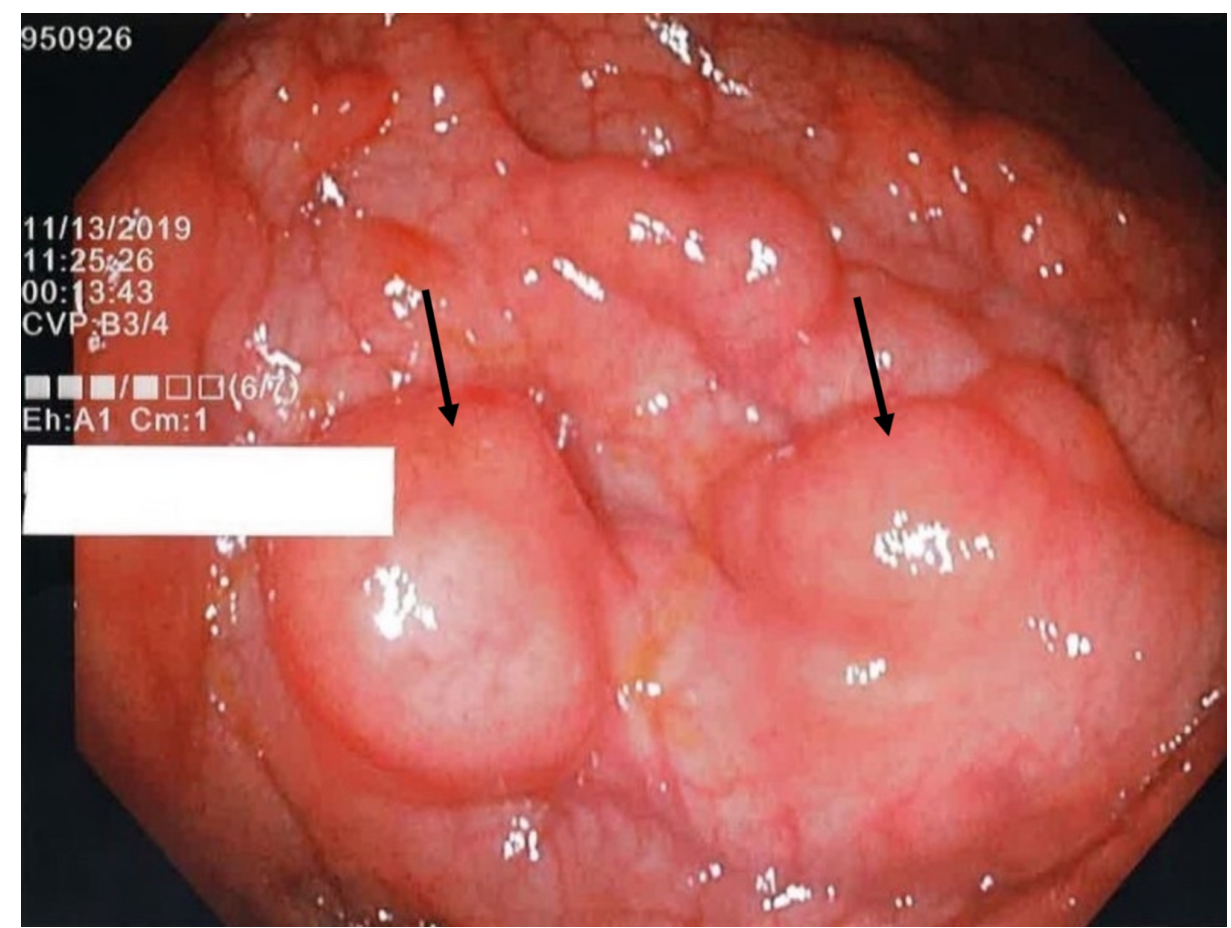

FIGURE 1: Colonoscopy findings of multiple masses at the splenic flexure consistent with $\mathrm{PCI}$

PCI: pneumatosis cystoides intestinalis; black arrows: cystic lesions arising from colonic mucosa

Biopsy of one of the grape-like masses at the splenic flexure was performed resulting in its visible rupture and collapse. Histologic examination of the biopsy demonstrated empty, submucosal cysts lined by multinucleated giant cells (Figure 2).

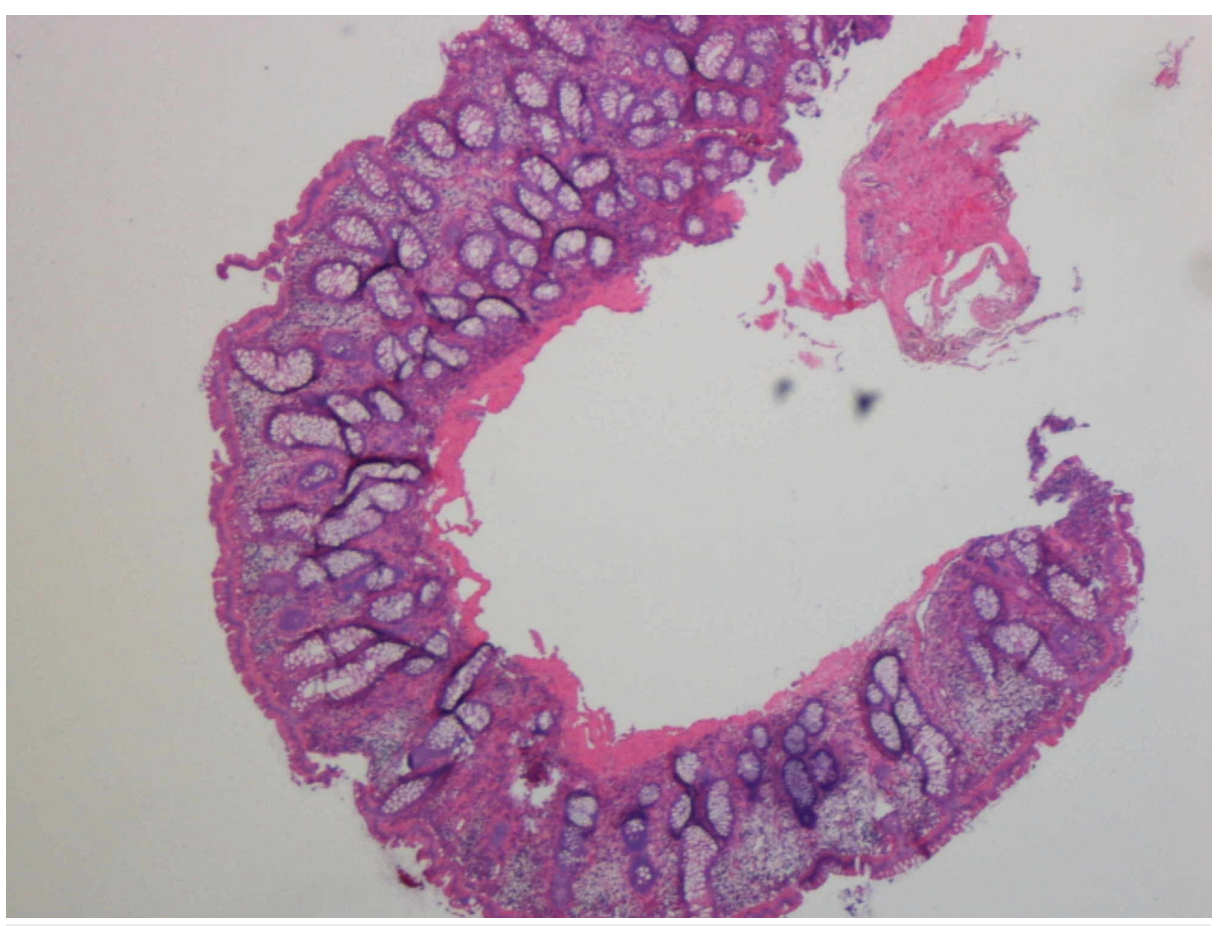

FIGURE 2: Histologic findings of submucosal cysts lined by multinucleated giant cells on sigmoid biopsy 


\section{Cureus}

Post-procedure CT images showed multiple, thin-walled, air-filled submucosal cysts with evidence of intraperitoneal free air (Figure 3). Given the patient was vitally stable without guarding or rigidity on exam, the decision was made to manage the patient conservatively with monitoring and serial exams. She remained asymptomatic and did not require surgical intervention. She was discharged home and has remained asymptomatic after three months of follow-up.

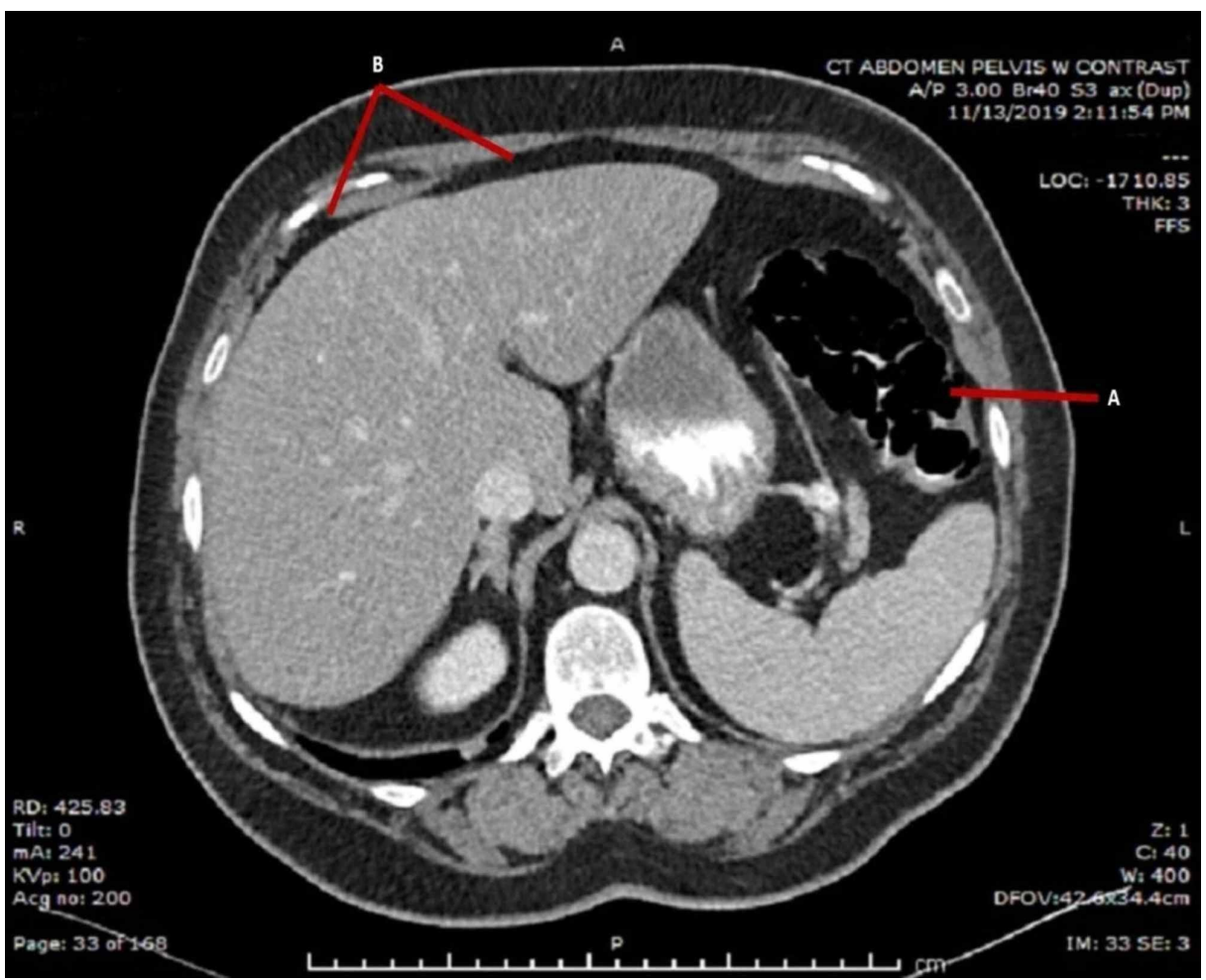

FIGURE 3: Post-colonoscopy CT abdomen revealing findings associated with pneumatosis cystoides intestinalis $(\mathrm{PCl})$ and pneumoperitoneum

(A) Air-filled cystic spaces within the gastrointestinal wall suggesting PCI; (B) findings of intra-abdominal free air.

\section{Discussion}

The pathogenesis of PCI remains incompletely understood, despite several hypotheses. These hypotheses suggest mucosal damage in combination with increased intraluminal pressure secondary to mechanical causes, bacterial production, or pulmonary gas traveling along the perivascular spaces of mesenteric vessels $[1,5,6]$. Approximately $15 \%$ of PCI cases have been categorized as idiopathic, or primary, in origin, while up to $85 \%$ of cases are secondary [7]. Disorders associated with PCI include, but are not limited to, chronic pulmonary disease, acquired immunodeficiency, history of transplant, systemic lupus erythematosus, inflammatory bowel disease, intestinal ischemia, and trauma [8]. Many patients with PCI are asymptomatic but can present with abdominal discomfort, diarrhea, abdominal distension, nausea, vomiting, hematochezia, mucousy stool, and constipation [9]. Our patient was asymptomatic and had not been diagnosed with any known associated diseases, though she does have a 46 pack-year history of tobacco abuse. It may be reasonable to hypothesize a subclinical respiratory disorder that may have contributed to her development of PCI, yet the true cause remains unknown.

Identification of PCI may be increasing along with the use of CT in diagnosing abdominal pathology [5]. CT is the ideal imaging modality for diagnosis of PCI, and a classic finding includes intramural gas parallel to the bowel wall (Figure 3) [10,11]. Prior reviews had found PCI to more commonly affect the small intestine, but more recent studies have found a higher incidence in the colon (46\%-61.8\%), as was seen with our patient [12]. The small intestine is affected in $15.4 \%-27 \%$ of cases, and only in $2.9 \%-7.0 \%$ cases is PCI present in both the colon and small intestine $[9,13,14]$.

Difficulty identifying patients with PCI secondary to a potentially deadly pathologic cause remains a barrier to management. A 2013 review found PCI to represent a benign finding in 60\% of cases and was associated with significant morbidity and mortality in approximately $40 \%$ of cases. Their analysis found a lactate elevation of 2.0 or greater to be the strongest independent predictor for pathologic disease. This lactate elevation in combination with hypotension or vasopressor need was found to have a predictive probability of 
93.2\% for pathologic disease [5]. Lactate $\geqslant 2.0$ at time of diagnosis has further been equated with a greater than $80 \%$ mortality rate, indicating a need for immediate surgical consultation and management [15].

Pneumoperitoneum, or free air in the abdominal cavity, is a concerning imaging finding that prompts immediate attention. It is estimated that $85 \%-95 \%$ of cases of pneumoperitoneum are associated with perforation of intra-abdominal viscus, and thus the tendency towards surgical management. In contrast, pneumoperitoneum found in association with PCI is often a benign finding but does not rule out viscus perforation [4]. This patient did undergo a colonoscopy, and while iatrogenic colonic perforation during colonoscopy is a possible explanation for the presence of pneumoperitoneum, it is quite rare with an estimated incidence of $0.12 \%$ [16]. Benign pneumoperitoneum following colonoscopy is thought to be rare or nonexistent with an estimated incidence of $0 \%-3 \%$ [17].

\section{Conclusions}

As the number of screening colonoscopies continues to rise, recognition of PCI by experienced endoscopists will become increasingly important. Identification can avoid misdiagnosis of polyposis or neoplasm and reduce unnecessary additional diagnostic studies while also ensuring proper management and follow-up to rule out pathologic causes. Although it is uncommon, clinicians and radiologists should also be on the lookout for PCI as a cause of benign pneumoperitoneum. Asymptomatic patients without concerning findings on exam may be managed conservatively with serial exams and avoid the complications and costs associated with surgical exploration or additional endoscopic procedures.

\section{Additional Information \\ Disclosures}

Human subjects: Consent was obtained by all participants in this study. Conflicts of interest: In compliance with the ICMJE uniform disclosure form, all authors declare the following: Payment/services info: All authors have declared that no financial support was received from any organization for the submitted work. Financial relationships: All authors have declared that they have no financial relationships at present or within the previous three years with any organizations that might have an interest in the submitted work. Other relationships: All authors have declared that there are no other relationships or activities that could appear to have influenced the submitted work.

\section{References}

1. St Peter SD, Abbas MA, Kelly KA: The spectrum of pneumatosis intestinalis . Arch Surg. 2003, 138:68-75. 10.1001/archsurg.138.1.68

2. Heng Y, Schuffler MD, Haggitt RC, Rohrmann CA: Pneumatosis intestinalis: a review. Am J Gastroenterol. 1995, 90:1747-1758.

3. Gui X, Zhou Y, Eidus L, Falck V, Gao Z-h, Qin L: Is pneumatosis cystoides intestinalis gas-distended and ruptured lymphatics?: Reappraisal by immunohistochemistry. Arch Pathol Lab Med. 2014, 138:1059-1066. 10.5858/arpa.2013-0145-OA

4. Mularski RA, Sippel JM, Osborne ML: Pneumoperitoneum: a review of nonsurgical causes. Crit Care Med. 2000, 28:2638-2644. 10.1097/00003246-200007000-00078

5. DuBose JJ, Lissauer M, Maung AA, et al.: Pneumatosis Intestinalis Predictive Evaluation Study (PIPES): a multicenter epidemiologic study of the Eastern Association for the Surgery of Trauma. J Trauma Acute Care Surg. 2013, 75:15-23. 10.1097/TA.0b013e318298486e

6. Galandiuk S, Fazio VW: Pneumatosis cystoides intestinalis. a review of the literature . Dis Colon Rectum. 1986, 29:358-363. 10.1007/bf02554132

7. Koss LG: Abdominal gas cysts (pneumatosis cystoides intestinorum hominis); an analysis with a report of a case and a critical review of the literature. AMA Arch Pathol. 1952, 53:523-549.

8. Knechtle SJ, Davidoff AM, Rice RP: Pneumatosis intestinalis. Surgical management and clinical outcome . Ann Surg. 1990, 212:160-165. 10.1097/00000658-199008000-00008

9. Wu LL, Yang YS, Dou Y, Liu QS: A systematic analysis of pneumatosis cystoids intestinalis . World J Gastroenterol. 2013, 19:4973-4978. 10.3748/wjg.v19.i30.4973

10. Feuerstein JD, White N, Berzin TM: Pneumatosis intestinalis with a focus on hyperbaric oxygen therapy . Mayo Clinic Proc. 2014, 89:697-703. 10.1016/j.mayocp.2014.01.026

11. Lassandro F, Valente T, Rea G, Lassandro G, Golia E, Brunese L, Laghi A: Imaging assessment and clinical significance of pneumatosis in adult patients. Radiol Med. 2015, 120:96-104. 10.1007/s11547-014-0461-5

12. Jamart J: Pneumatosis cystoides intestinalis. A statistical study of 919 cases . Acta Hepatogastroenterol. 1979, 26:419-422.

13. Horiuchi A, Akamatsu T, Mukawa K, Ochi Y, Arakura N, Kiyosawa K: Case report: pneumatosis cystoides intestinalis associated with post-surgical bowel anastomosis: a report of three cases and review of the Japanese literature. J Gastroenterol Hepatol. 1998, 13:534-537. 10.1111/j.1440-1746.1998.tb00682.x

14. Morris MS, Gee AC, Cho SD, Limbaugh K, Underwood S, Ham B, Schreiber MA: Management and outcome of pneumatosis intestinalis. Am J Surg. 2008, 195:679-683. 10.1016/j.amjsurg.2008.01.011

15. Hawn MT, Canon CL, Lockhart ME, Gonzalez QH, Shore G, Bondora A, Vickers SM: Serum lactic acid determines the outcomes of CT diagnosis of pneumatosis of the gastrointestinal tract. Am Surg. 2004, 70:19-23.

16. Lüning TH, Keemers-Gels ME, Barendregt WB, Tan ACITL, Rosman C: Colonoscopic perforations: a review of 30,366 patients. Surg Endosc. 2007, 21:994-997. 10.1007/s00464-007-9251-7 


\section{Cureus}

17. Pearl JP, McNally MP, Elster EA, DeNobile JW: Benign pneumoperitoneum after colonoscopy: a prospective pilot study. Mil Med. 2006, 171:648-649. 10.7205/milmed.171.7.648 